\title{
CARACTERIZAÇÃO DE SUBSTÂNCIAS HÚMICAS EXTRAÍDAS DE SOLOS E DE LODO ORGÂNICO(1)
}

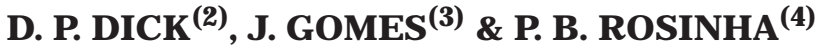

\begin{abstract}
RESUMO
No presente trabalho, foi estudada a extração de substâncias húmicas (SH) de horizontes A de dois solos do Rio Grande do Sul (Latossolo Roxo (LR), e Brunizém vértico (B)) e de um lodo orgânico (LO), oriundo da fabricação de celulose, empregando-se dois métodos: (1) as SH foram extraídas com solução de $\mathrm{NaOH}$ 0,5 mol L-1, após tratamento prévio com HCl 0,5 mol L-1; (2) foi introduzida a extração com solução de pi rofosfato de sódio $0,15 \mathrm{~mol} \mathrm{~L}^{-1}$ (pH 7) antes da etapa alcalina. Em cada etapa, os teores de SH, de ácidos fúlvicos (AF) e de ácidos húmi cos (AH) foram determinados por métodos espectroscópicos e gravimétricos e, no método 2, os teores de Fe, Al, Si e Ca foram medidos nos extratos por absorção atômica. A composição elementar de ácidos húmicos foi determinada, e sua estrutura e características químicas foram avaliadas a partir de razões $\mathbf{C} / \mathrm{N}$, (atômicas) H/C e O/C e a partir de dados de espectroscopia Ultravioleta/Visível (UV/Nis). No LR, o método 1 extraiu maior quantidade de SH, enquanto, no método 2, a etapa com pirofosfato extraiu apenas AF. Em contrapartida, no solo B, o método 2 foi mais eficiente, verificando-se maior rendimento na etapa com pirofosfato. A partir desses resultados, infere-se que as SH, no LR, interagem principal mente por meio de reação de trocas de ligantes e de pontes de hidrogênio e, no solo B, a interação por meio de pontes de cátions é um mecanismo importante. Os AH extraídos com $\mathrm{NaOH}$ apresentaram valores mais elevados para a razão $\mathrm{H} / \mathrm{C}$ e valores inferiores para a razão $\mathrm{O} / \mathrm{C}$ do que os extraídos com pirofosfato, indicando que o extrator neutro solubilizou SH de caráter aromático e grau de oxigenação superiores.
\end{abstract}

Termos de indexação: extração neutra, extração alcalina, espectroscopia UVNis.

\footnotetext{
(1) Trabalho desenvolvido com apoio do CNPq, e apresentado parcialmente no XXVI Congresso Brasileiro de Ciência do Solo, Rio de J aneiro, 20 a 26 de julho de 1997. Recebido para publicação em abril e aprovado em setembro de 1998.

(2) Professor Adjunto do Instituto de Química, Universidade Federal do Rio Grande do Sul - UF RGS. Av. Bento Gonçalves, 9500, CE P 91501-970 Porto Alegre (RS).E-mail: DPDICK @if1.if.ufrgs.br. Bolsista do CNPq. Professor do CPG em Ciência do Solo, Faculdade de Agronomia, UFRGS.

(3) Aluna de graduação do curso de Bacharelado em Química, UFRGS. Bolsista de IC da FAPERGS.

(4) Aluna de graduação do curso de Engenharia Química, UFRGS. Bolsista de IC do CNPq.
} 


\title{
SUMMARY: CHARACTERIZATION OF HUMIC SUBSTANCES EXTRACTED FROM SOILS AND ORGANIC SLUDGE
}

\begin{abstract}
The extraction of humic substances (HS) from A-horizons of two soils from Rio Grande do Sul (Oxisol, LR, and Mollisol, BZ) and from an organic sludge (LO) was investigated, employing two methods: (1) the HS wereextracted with $0.5 \mathrm{~mol} \mathrm{~L}^{-1} \mathrm{NaOH}$, after treating the sample with $0.5 \mathrm{~mol} \mathrm{~L}^{-1} \mathrm{HCl}$; (2) the extraction with $0.15 \mathrm{~mol} \mathrm{~L}^{-1}$ sodium pyrophosphate solution ( $\mathrm{pH}$ 7) was introduced before the alkal ine and after the acid treatments. In each step, the contents of HS, fulvic acids (FA) and humic acids (HA) were determined by spectroscopic and gravimetric methods, and the contents of co-extracted Fe Al, Si and Ca in theextracts of method 2 weremeasured by atomic absorption. The lemental compositi on of $\mathrm{HA}$ was determined and their chemical structureand characteristics wereevaluated from the $\mathrm{C} / \mathrm{N}, \mathrm{H} / \mathrm{C}$ and $\mathrm{O} / \mathrm{C}$ rati os and from UV/ Vis spectroscopy data. I n the Oxi sol, method 1 extracted a greater amount of $\mathrm{HS}$, and in method 2 the pyrophosphate step extracted only AF. On the other hand, method 2 was more efficient in the Mollisol, and the greatest yiedd was obtained in the pyrophosphate step. These results suggest that in the Oxisol, the HS interact mainly through ligand exchange reactions and hydrogen bonding, while in the $\mathrm{Mol}$ lisol, cation bridges arealso an important mechanism. The $\mathrm{NaOH}$-extracted $\mathrm{HA}$ showed higher $\mathrm{H} / \mathrm{C}$ and lower $\mathrm{O} / \mathrm{C}$ ratios than the pyrophosphate-extracted ones, indicating that theneutral solution solubilized HS with higher aromatic character and oxygenation grade
\end{abstract}

Index terms: neutral extraction, alkal ine extraction, UV/ Vis spectroscopy.

\section{INTRODUÇÃO}

As substâncias húmicas (SH) são encontradas em sol os nas seguintes formas: (a) comoagregados macromoleculares insolúveis; (b) como macromoléculas ligadas entre si por meio de pontes de cátions di- e trivalentes; (c) associadas aos minerais por meio de pontes catiônicas, pontes de hidrogênio e reações de troca de ligantes, e (d) oclusas nas entrecamadas de argilominerais expansivos (Stevenson, 1994).

Em óxidos de ferro sintéticos, observou-se que a troca deligantes entre grupos funcionais carboxílicos e hidroxílicos da matéria orgânica com a superfície do mineral foi o mecanismo de interação dominante (Gu et al., 1994). Anteriormente, Parfitt et al . (1977) haviam observado que as $\mathrm{SH}$ podem interagir com gibbsita eóxidos de ferro tanto via troca de ligantes como via pontes de hidrogênio. O mecanismo de pontes de cátions pode tornar-se extremamente importante para a fixação de SH em superfícies laterais de caulinita e nas basais de ilita (Varadachari et al., 1995), enquanto em esmectitas a interação de $\mathrm{SH}$ e polímeros de hidroxi-Al nas entrecamadas foi relatada como sendo de natureza forte, uma vez que a remoção da matéria orgânica não foi completa após tratamento com percl orato de sódio (Singer \& Huang, 1993). A interação por meio de pontes dehidrogênio deSH ecaulinita, esmectitas e ilita foi constatada por meio de técnicas espectroscópicas por Ristori et al. (1992).
O método de extração de SH mais amplamente empregado e citado como o mais eficiente consiste no tratamento com solução de $\mathrm{NaOH}$ 0,1 a 0,5 mol L-1 (Senesi et al., 1994; Stevenson, 1994), sendotambém recomendado pela Sociedade Internacional de Substâncias Húmicas (Calderoni \& Schnitzer, 1984). Senesi et al. (1994) alertam que extrações consecutivas e de longa duração podem modificar as SH extraídas, no entanto, estudos sistemáticos mostraram que $\mathrm{SH}$ extraídas com $\mathrm{NaOH}$ durante $24 \mathrm{~h}$ em presença do ar não sofreram oxidação (Tan et al., 1991). A extração deSH por meio deste método é verificada primordialmente por dois mecanismos: (a) rompimento de pontes de hidrogênio entre as moléculas orgânicas entre si e com a superfície do mineral, e (b) reação de troca de ligantes entre as hidroxilas do meio e os grupos carboxílicos efenól icos da molécula da substância húmica, pelos quais a substância húmica está coordenada na superfície hidroxilada do mineral (Stevenson, 1994; Cornejo \& Hermosín, 1996).

Um método de extração mais brando e sel etivo emprega solução de $\mathrm{Na}_{4} \mathrm{P}_{2} \mathrm{O}_{7} 0,15$ mol L-1, podendo, no entanto, ser menos eficiente do que o alcalino (Senesi et al., 1994; Swift, 1996). O mecanismo principal de extração de SH desse método consiste no rompimento das pontes catiônicas que unem as moléculas de $\mathrm{SH}$ entre si e, ou, com a superfície do mineral, decorrente da complexação do ânion pirofosfato com cátions di- etrival entes participantes 
dessetipo de interação (Stevenson, 1994; Cornejo \& Hermosin, 1996). Hayes et al. (1996), ao fracionar ácidos húmicos $(\mathrm{AH})$ e ácidos fúlvicos $(\mathrm{AF})$ de um Mollisol com soluções de pirofosfato em diferentes níveis de $\mathrm{pH}$, constataram que as $\mathrm{SH}$ extraídas em pH 7,0 eram mais aromáticas, enquanto as extraídas em $\mathrm{pH}$ alcalino apresentavam maior teor de carboidratos.

No presente estudo, investigou-se o efeito da extração com solução neutra de pirofosfato na extração al calina de substâncias húmicas num solo com predomínio de carga permanente (Brunizém vértico) e num solo onde predominam cargas variáveis (Latossolo Roxo). A fim de avaliar a influência da presença da fração mineral do solo sobre a solubilização de $\mathrm{SH}$, analisou-se um lodo orgânico.

\section{MATERIAL E MÉTODOS}

As substâncias húmicas extraíveis (SH), incluindo os ácidos húmicos $(A H)$ e fúlvicos $(A F)$, foram solubilizadas de amostras compostas do horizonte A (0 a $10 \mathrm{~cm}$ ) de dois solos (Quadro 1) e de um lodo orgânico oriundo da fabricação de celulose (Dick et al., 1997). O Brunizém (B) encontrava-se sob campo nativo e o Latossolo Roxo(LR) sob lavoura (sucessão aveia-soja).

\section{Fracionamento das substâncias húmicas}

Nos testes de extração e fracionamento das $\mathrm{SH}$, foram empregados $3 \mathrm{~g}$ de solo e $0,7 \mathrm{~g}$ de lodo com duas repetições. As médias foram comparadas pelo Teste de Duncan a $5 \%$.

Método 1 (adaptado de Swift, 1996): A amostra foi tratada três vezes com $30 \mathrm{ml}$ de $\mathrm{HCl}$ 0,5 mol L-1 sob agitação durante 2 h. O sobrenadante foi levado a um volume conhecido e reservado para análises posteriores. A seguir, procedeu-se à extração com $30 \mathrm{ml}$ de NaOH 0,5 mol L-1 ao ar com 3 h de agitação (5 vezes). O sobrenadante foi filtrado em papel analítico quantitativo para eliminar a argila que não sedimentou com a centrifugação (10 min, 2.000 rpm) e completou-se a um volume conhecido. Após a retirada de uma alíquota de $15 \mathrm{ml}$ para análises, adicionou-se $\mathrm{HCl}$ 0,1 mol L-1 até $\mathrm{pH} 2$ e deixou-se o sistema em repouso durante $24 \mathrm{~h}$. Separou-se o AH precipitado por centrifugação, lavou-se o material com $30 \mathrm{ml}$ de solução de $\mathrm{HF} / \mathrm{HCl} 5 \%(3 \times 2 \mathrm{~h})$ para eliminar os contaminantes inorgânicos. A seguir, o $\mathrm{AH}$ suspenso em água destilada foi congelado durante uma noite e, após o descongelamento, foi lavado com água destilada até teste negativo com $\mathrm{AgNO}_{3}$ para íons cloreto. O AH purificado foi seco em estufa a vácuo a $60^{\circ} \mathrm{C}$ em um frasco pré-pesado. $\mathrm{O}$ teor de $\mathrm{AH}$ foi determinado, relacionando-se sua massa seca com a da amostra total, após descontado o teor de cinzas. Esta variável foi determinada por cal cinação a $750^{\circ} \mathrm{C}$ durante quatro horas em mufla. A solução de AF foi levada a um volume conhecido e retirou-se uma alíquota de $20 \mathrm{ml}$ para análises.

Método 2 (adaptado de Swift, 1996): Neste método, após o tratamento com $\mathrm{HCl} 0,5$ mol L-1, a amostra foi submetida à extração com $30 \mathrm{ml}$ de solução de $\mathrm{Na}_{4} \mathrm{P}_{2} \mathrm{O}_{7} 0,15$ mol L-1, pH 7,0 (5 x 2 h). Em seguida, realizou-se a extração com $\mathrm{NaOH} 0,5 \mathrm{~mol}$ L-1 (3 $\times 3$ h). Na separação dos sobrenadantes e da fração de $\mathrm{AH}$, procedeu-se conforme descrito no método 1 .

\section{Espectroscopia na região UVNis}

Nos sobrenadantes de SH e deAF, foi determinado o espectro de 200 a 800 nm (Espectrofotômetro Shimadzu UV-160A). N os extratos de SH extraídas com pirofosfato ( $\mathrm{pH}$ 7) edeAF ( $\mathrm{pH} 2), \mathrm{opH}$ foi elevado para 13, com vistas em comparar os resultados com os do extrato al cal ino. Foi calculada a razão entre as absorbâncias a 350 e 550 nm ( $\left.E_{350} / E_{550}\right)$, adaptandose o método de Burba et al. (1995). Calculou-se, adicional mente, um índice de quantidade para SH e $\mathrm{AF}$, estimado a partir da diferença $\mathrm{E}_{450}-\mathrm{E}_{700}$. $\mathrm{Na}$ obtenção desta variável, multiplicou-se o valor da diferença entre as absorbâncias pel o fator de diluiçã̃o (extratos de pirofosfato e deAF) e pel o vol ume total em $\mathrm{ml}$, e dividiu-se pela massa. A partir dos valores corrigidos, calcul ou-se a razão espectrocópica AF/SH.

\section{Carbono orgânico}

Nos extratos de $\mathrm{SH}$ e de AF, foi determinado o teor de carbono, medindo-sea absorbância da solução a $580 \mathrm{~nm}$ após reação durante $4 \mathrm{~h}$ a $60^{\circ} \mathrm{C}$ com solução ácida de dicromato de potássio ( $3 \mathrm{ml}: 3 \mathrm{ml}$ ) (Tinsley, 1950). A curva-padrão foi construída com glicose de 0 a 200 mg L-1. As quantidades deSH eAF extraídas

Quadro 1. Classificação dos solos e localização dos pontos de coleta das amostras

\begin{tabular}{ccccc}
\hline Amostra & Símbolo & Mineralogia da fração argila & C orgânico & Local de coleta \\
\hline & & & $\mathrm{g} \mathrm{kg}^{-1}$ & \\
& $\mathrm{HR}$ & $\mathrm{Hm}, \mathrm{Gt}, \mathrm{Ka}$ & 26,8 & Santo Ângelo, RS \\
Latossolo Roxo* (Oxisol***) & $\mathrm{LR}$ & 21,1 & Pantano Grande, RS \\
Brunizem vértico** $\left(\mathrm{Mollisol}{ }^{* * *}\right)$ & $\mathrm{B}$ & $\mathrm{Ka}$ & 21 & \\
\hline
\end{tabular}

* Bayer (1996); **Albuquerque (1998). *** Soil Taxonomy; Hm: hematita; Gt: goethita; Ka: caulinita; 2:1:argilominerais 2:1. 
foram estimadas em função dos teores de carbono determinados nos respectivos sobrenadantes, considerando ser o carbono em solução em cada extrato parteintegrante exclusivamentede $\mathrm{SH}\left(\mathrm{C}_{\mathrm{SH}}\right)$ e de $A F\left(C_{A F}\right)$, respectivamente. Os valores determinados em solução foram reportados à massa inicial de solo extraída: $\mathrm{C}_{\mathrm{SH}}=\mathrm{mg} \mathrm{C}$ na forma de $\mathrm{SH} / \mathrm{g}$ de solo; $C_{A F}=m g C$ na forma de AF/g de solo.

\section{Elementos coextraídos}

Os elementos $\mathrm{Fe}, \mathrm{Al}$, Si e Ca extraídos nos sobrenadantes de $\mathrm{HCl}$, de pirofosfato e de $\mathrm{NaOH}$ do método 2 foram determinados por espectrofotometria de absorção atômica (Especrofotômetro Perkin Elmer 403).

\section{Análise elementar}

Nos AH purificados, foram determinados os teores de $\mathrm{C}, \mathrm{H}$ e $\mathrm{N}$ (analisador elementar Perkin Elmer 4.000) e, após correção destes val ores para baselivre decinzas, foi caculado oteor de oxigênio por diferença de $1.000 \mathrm{mg} \mathrm{g}^{-1}$.

\section{RESULTADOS E DISCUSSÃO}

\section{Quantidades de substâncias húmicas extraídas}

As quantidades de $\mathrm{SH}$ e deAF, avaliadas segundo o teor de carbono determinado nos respectivos extratos e cal culado para cada fração $\left(\mathrm{mg} \mathrm{g}^{-1}\right.$ de C no solo), variaram com o tipo de agente extrator nas três amostras analisadas (Quadro 2).
No LR, o método 1 extraiu mais $\mathrm{SH}$ do que o método 2 (Quadro 2). A etapa com pirofosfato deste último não extraiu $\mathrm{AH}$ e, aparentemente, teve um efeito supressor na extração subseqüente de $\mathrm{AH}$ com $\mathrm{NaOH}$. Em contrapartida, $\mathrm{C}_{\mathrm{AF}}$ foi superior no método 2. Consi derando os mecanismos de sol ubilização dos agentes extratores empregados, infere-se que, neste solo, os $\mathrm{AH}$ reagiram principalmente com as superfícies dos minerais por meio de pontes de hidrogênio etroca deligantes, enquanto os AF também interagiram por meio de pontes catiônicas.

NoB, as médias de $\mathrm{C}_{\mathrm{SH}}$ no método 1 eno método 2 não se mostraram diferentes estatisticamente, porém foram observados val ores mais elevados para $\mathrm{C}_{\mathrm{AF}}$ eAH do método 2, o queindiretamente corrobora a diferença entre os valores de $\mathrm{C}_{\mathrm{SH}}$. A etapa com pirofosfato extraiu, aproximadamente, 55\% dos teores totais deAH e deAF, indicando a importância do mecanismo de pontes catiônicas neste solo com predomínio de cargas permanentes. Resultados semel hantes foram obtidos por Hayes et al. (1996) com extrações sucessivas em diferentes níveis de pH em um Mollisol. Adicional mente, os maiores val ores obtidos para $C_{A F}$ pelo método 2 na amostra $B$ evidenciam que o agente complexante pirofosfato extraiu substâncias não-suscetíveis à extração alcalina.

No LO, os valores obtidos para $\mathrm{C}_{\mathrm{SH}}$ e $\mathrm{AH}$ pelo método 1 não se diferenciaram estisticamente daqueles obtidos pelo método 2. J á os teores de AF foram superiores para o método 2 (Quadro 2), ocorrendo o mesmo comportamento observado para o solo B. Nesta amostra, ondenão existem minerais, pode-se inferir que tanto as pontes catiônicas como as pontes de hidrogênio constituem mecanismos de interação importantes entre as moléculas de SH.

Quadro 2. Teores de carbono nos extratos de $\mathrm{SH}$ e de $\mathrm{AF}$, razão $\mathrm{C}_{\mathrm{AF}} / \mathrm{C}_{\mathrm{SH}}$, razão $\mathrm{AF} / \mathrm{SH}$ obtida dos dados de UV/Nis, e teores de AH nas amostras, segundo os métodos de extração 1 e 2

\begin{tabular}{|c|c|c|c|c|c|c|c|}
\hline Amostra & Método & Etapa & $\mathrm{C}_{\mathrm{SH}}$ & $\mathrm{C}_{\mathrm{AF}}$ & $\mathrm{C}_{\mathrm{AF}} / \mathrm{C}_{\mathrm{SH}}$ & AF/SH (UV/Vis) & $\mathbf{A H}$ \\
\hline \multirow[t]{2}{*}{ LR } & $\begin{array}{l}1 \\
2\end{array}$ & $\begin{array}{l}\mathrm{NaOH} \\
\text { Pirofosfato } \\
\mathrm{NaOH}\end{array}$ & $\begin{array}{l}\frac{6,1}{a} \\
1,8 \mathrm{~d} \\
3,1 \mathrm{c}\end{array}$ & $\begin{array}{l}\frac{3,0}{2,0} \mathrm{ab} \\
1,6 \mathrm{c}\end{array}$ & $\begin{array}{l}\frac{0,48}{c} \\
1,10 \mathrm{a} \\
0,52 \mathrm{c}\end{array}$ & $\begin{array}{l}0,34 \mathrm{~b} \\
0,71 \mathrm{a} \\
0,24 \mathrm{~b}\end{array}$ & $\begin{array}{l}2,9 \mathrm{a} \\
0,0 \mathrm{c} \\
2,3 \mathrm{~b}\end{array}$ \\
\hline & & Total & $\underline{4,9 \mathrm{~b}}$ & $\underline{3,6}$ a & $\underline{0,73} \mathrm{~b}$ & n.d. & $\underline{2,3} \mathrm{~b}$ \\
\hline \multirow[t]{2}{*}{ B } & $\begin{array}{l}1 \\
2\end{array}$ & $\begin{array}{l}\mathrm{NaOH} \\
\text { Pirofosfato } \\
\mathrm{NaOH}\end{array}$ & $\begin{array}{c}\frac{11,2 \mathrm{ab}}{8,6 \mathrm{bc}} \\
5,9 \mathrm{c}\end{array}$ & $\begin{array}{l}\frac{4,3}{3} \mathrm{~b} \\
3,3 \mathrm{bc} \\
2,7 \mathrm{c}\end{array}$ & $\begin{array}{l}\frac{0,38}{0} a \\
0,39 a \\
0,46 a\end{array}$ & $\begin{array}{l}\frac{0,12}{b} \\
0,38 \mathrm{a} \\
0,11 \mathrm{~b}\end{array}$ & $\begin{array}{l}\frac{7,5}{b} \mathrm{~b} \\
6,4 \mathrm{~b}\end{array}$ \\
\hline & & Total & $\underline{14,5} \mathrm{a}$ & $\underline{6,1} a$ & $\underline{0,42} \mathrm{ab}$ & n.d & $\underline{13,8} \mathrm{a}$ \\
\hline \multirow[t]{2}{*}{ LO } & $\begin{array}{l}1 \\
2\end{array}$ & $\begin{array}{l}\mathrm{NaOH} \\
\text { Pirofosfato } \\
\mathrm{NaOH}\end{array}$ & $\begin{array}{l}74,2 \mathrm{a} \\
27,3 \mathrm{~b} \\
41,0 \mathrm{~b}\end{array}$ & $\begin{array}{l}\frac{15,7}{15} \\
10,0 \mathrm{c} \\
10,6 \mathrm{c}\end{array}$ & $\begin{array}{l}\frac{0,21}{b} \\
0,36 \mathrm{a} \\
0,26 \mathrm{~b}\end{array}$ & $\begin{array}{l}0,24 a \\
0,32 a \\
0,24 a\end{array}$ & $\begin{array}{r}110,1 \mathrm{a} \\
41,8 \mathrm{~b} \\
51,2 \mathrm{~b}\end{array}$ \\
\hline & & Total & $\underline{68,3}$ a & $\underline{20,7}$ a & $\underline{0,30} a b$ & n.d. & $\underline{93,0}$ a \\
\hline
\end{tabular}

n.d.: não-determinável. $\mathrm{C}_{\mathrm{SH}}=\mathrm{mg} \mathrm{C}$ na forma de $\mathrm{SH} / \mathrm{g}$ de solo; $\mathrm{C}_{\mathrm{AF}}=\mathrm{mg} \mathrm{C}$ na forma de $\mathrm{AF} / \mathrm{g}$ de solo; $\mathrm{AH}=\mathrm{mg} \mathrm{AH} / \mathrm{g}$ de solo. Letras minúsculas, na coluna, diferenciam médias, pelo teste de Duncan a $5 \%$, para cada amostra. 
É conveniente salientar que os valores de $\mathrm{C}_{\mathrm{SH}} \mathrm{e}$ de $C_{A F}$ não devem ser comparados em termos absolutos com os valores de teor de $\mathrm{AH}$, uma vez quesão variáveis diferentes queforam determinadas por métodos distintos.

Os valores da razão $\mathrm{C}_{\mathrm{AF}} / \mathrm{C}_{\mathrm{SH}}$ indicam que, na etapa com pirofosfato no método 2 , houve uma extração preferencial de $A F$ em relação a $A H$ nas amostras LR e LO (Quadro 2). Em contrapartida, na amostra $\mathrm{B}$, a etapa com $\mathrm{NaOH}$ do método 2 apresentou valor superior para a razão $\mathrm{C}_{\mathrm{AF}} / \mathrm{C}_{\mathrm{SH}}$. Tais resultados podem indicar que, neste solo, o pirofosfato, ao complexar metais, torna os ácidos fúlvicos mais labéis também para etapas posteriores, de maneira análoga, porém mais intensa, à ação do pré-tratamento com $\mathrm{HCl}$. Por outrolado, oval or al to de 1,1 para $C_{A F} / C_{S H}$, na etapa com pirofosfato do método 2 na amostra $L R$, sugere uma subestimação de $\mathrm{C}_{\mathrm{SH}}$. Este fato pode estar relacionado com a oxidação incompleta do carbono orgânico por parte do dicromato no sobrenadante de SH. A fração SH, que continha também $\mathrm{AH}$, pode ter apresentado maior complexidade químico-estrutural do que $A F$, o quea tornaria mais resistente à oxidação. Todavia, uma vez que os resultados do presente estudo estão sendo comparados entre as amostras, isto não afeta a discussão.

A razão AF/SH, obtida a partir de dados da espectroscopia UV/Vis, apresentou a mesma tendência de variação observada para $\mathrm{C}_{\mathrm{AF}} / \mathrm{C}_{\mathrm{SH}}$, podendo ser empregada eventualmente para fins comparativos. Todavia, nos solos, os valores de AF/ $\mathrm{SH}$ foram sempre inferiores aos de $\mathrm{C}_{\mathrm{AF}} / \mathrm{C}_{\mathrm{SH}}$, mostrando que outras variáveis podem influir na absorbância medida. Por exemplo, a fração argila dispersa simultaneamente com as substâncias húmicas, ou nelas adsorvida, contribuiria para maior valor da absorbância do extrato de $\mathrm{SH}$ e, conseqüentemete, diminuiria a razão $\mathrm{AF} / \mathrm{SH}$. No lodo, onde não existem minerais, os valores para os dois métodos foram muito próximos.

\section{Razão $\mathrm{E}_{\mathbf{3 5 0}} / \mathrm{E}_{\mathbf{5 5 0}}$}

A razão entre as absorbâncias do espectro na região UVNis é uma variável usual para estimar o grau de condensação estrutural e o tamanho médio da molécula das SH (Bloom \& Leenheer, 1989; Burba et al., 1995). Optou-se, no presente estudo, em determinar o espectro em $\mathrm{pH} 13$ contrariamente ao recomendado por Chen et al. (1977), uma vez que os extratos com a totalidade de $\mathrm{SH}$ foram analisados. Tal procedimento não influiu na qualidade dos resultados, pois se está fazendo um estudo comparativo entre amostras. Adicionalmente, em concentrações el evadas de el etrólitos, como foi o caso dos extratos empregados nas medidas de absorbâncias, as substâncias húmicas apresentam conformação de colói des esféricos rígidos (Ghosh \& Schnitzer, 1980), preenchendo os requisitos estabelecidos por Chen et al. (1977).
Quadro 3. Razão $E_{350} / E_{550}$ nos extratos de SH e de AF dos métodos 1 e 2

\begin{tabular}{|c|c|c|c|c|c|c|}
\hline \multirow{3}{*}{ Amostra } & \multicolumn{6}{|c|}{ Método } \\
\hline & \multicolumn{2}{|c|}{1 - NaOH } & \multicolumn{2}{|c|}{2 - Pirofosfato } & \multicolumn{2}{|c|}{ 2-NaOH } \\
\hline & SH & AF & SH & AF & SH & AF \\
\hline $\begin{array}{l}\text { LR } \\
\text { B } \\
\text { LO }\end{array}$ & $\begin{array}{l}6,8 \mathrm{~b} \\
4,1 \mathrm{e} \\
6,3 \mathrm{e}\end{array}$ & $\begin{array}{l}14,2 \mathrm{a} \\
10,0 \mathrm{a} \\
19,0 \mathrm{~b}\end{array}$ & $\begin{array}{l}12,7 \mathrm{a} \\
5,9 \mathrm{~d} \\
8,3 \mathrm{~d}, \mathrm{e}\end{array}$ & $\begin{array}{r}13,8 \mathrm{a} \\
7,4 \mathrm{c} \\
11,1 \mathrm{c}\end{array}$ & $\begin{array}{l}7,1 \mathrm{~b} \\
4,2 \mathrm{e} \\
9,5 \mathrm{c}, \mathrm{d}\end{array}$ & $\begin{array}{r}11,2 \mathrm{a} \\
9,0 \mathrm{~b} \\
28,0 \mathrm{a}\end{array}$ \\
\hline
\end{tabular}

Letras minúsculas, na linha, diferenciam médias, pelo teste de Duncan a $5 \%$.

Em ambos os solos, a razão $\mathrm{E}_{350} / \mathrm{E}_{550}$ de $\mathrm{SH}$ do extrato alcalino no método 1 não diferiu significativamente do val or obtido para este extrato no método 2, indicando que substâncias estrutural e quimicamente semel hantes foram extraídas (Quadro 3). Na amostra LR, os valores de $\mathrm{E}_{350} / \mathrm{E}_{550}$ nos extratos de pirofosfato deSH eAF não diferiram, confirmando que, neste solo, somenteAF foi extraído nesta etapa. No B, os extratos de pirofosfato apresentaram valor superior para SH einferior para $A F$, quando comparados com os val ores dos extratos al calinos. Em relação ao AF, infere-se que a extração neutra solubilizou ácidos fúlvicos de mai or tamanho molecular médio.

Anal ogamente ao observado para as amostras de solo, na amostra $L O$, a razão $\mathrm{E}_{350} / \mathrm{E}_{550}$ observada para AF foi sempre maior do que a obtida para a fração SH. De acordo com Stevenson (1994), esteresultado não é surpreendente, pois indica queAF apresentou menor tamanho molecular e, ou, menor condensação estrutural do que a totalidade de $\mathrm{SH}$.

\section{Elementos solubilizados nos extratos do método 2}

Os teores de $\mathrm{Ca}$ extraído com $\mathrm{HCl}$ foram de $1,2 \mathrm{mg} \mathrm{g}^{-1}$ solo no LR, 2,3 $\mathrm{mg} \mathrm{g}^{-1}$ solo no $B$ e $8,4 \mathrm{mg} \mathrm{g}^{-1}$ no LO. Na extração neutra, os teores foram muito baixos ( $<0,7 \mathrm{mg} \mathrm{g}^{-1}$ solo) nas três amostras, devido, provavel mente, à preci pitação do pir ofosfato de cálcio (Stevenson, 1994). No extrato alcalino, os níveis de Ca detectados foram também muito baixos $\left(<0,3 \mathrm{mg} \mathrm{g}^{-1}\right)$, revelando que as formas de cálcio associadas à matéria orgânica haviam sido previamente extraídas pel os outros extratores. Por essa razão, os dados de Ca não foram reportados no gráficos.

Os teores de $\mathrm{Al}$ e de $\mathrm{Si}$ extraídos com $\mathrm{HCl}\left(\mathrm{Al}_{\mathrm{H}}\right.$ e $\mathrm{Si}_{\mathrm{H}}$ ) foram relativamente semel hantes nos sol os LR eB. (Figuras 1 e2). Os el ementos solubilizados nesta etapa são provavel mente oriundos não só de formas fracamente associadas com a matéria orgânica, formas trocáveis, mas também de formas mal cristalizadas de oxidróxidos. O tratamento prévio com áci do tem por objetivo romper pontes de cátions 
que, eventualmente, dificultariam a extração subseqüente das SH (Stevenson, 1994). O teor de $\mathrm{Fe}_{\mathrm{H}}$ foi maior no $\mathrm{B}$ do que no LR, e esta diferença pode estar associada à razão $\mathrm{Fe}_{\mathrm{d}} / \mathrm{Fe}_{\mathrm{d}}$ mais el evada para o Brunizém (0,23; Al buquerque (1998)) quando comparada com a do LR (0,10; Bayer (1996))

Os teores observados para $\mathrm{Fe}, \mathrm{Al}$ e $\mathrm{Si}$ extraídos com a solução neutra ( $F e_{p}, \mathrm{Al}_{\mathrm{p}}$ e $\mathrm{Si}_{\mathrm{p}}$ ) tenderam a valores maiores no B (Figura 2), quando comparados com os de LR (Figura 1), ainda que, em al guns casos, a diferença não tenha sido estatisticamente significativa. A extração com pirofosfato em pH básico $(9,5-10,5)$ é um método usualmente empregado para determinar as formas iônicas de Fe e deAl ligadas à matéria orgânica (Borggaard, 1988). No entanto, alguns autores mostraram que o $\mathrm{Fe}_{\mathrm{p}}$ pode ser também atribuído à goethita e ferridrita adsorvidas à matéria orgânica extraída ou mesmo dispersas no meio levemente básico (Schuppli et al., 1983; Parfitt \& Childs, 1988). Quanto ao alumínio, Kaiser \& Zech (1996) constataram que os teores solubilizados pela extração com pirofosfato são devidos também à dissolução alcalina de fases hidróxidas e de hidróxidos adsorvidos à matéria orgânica.

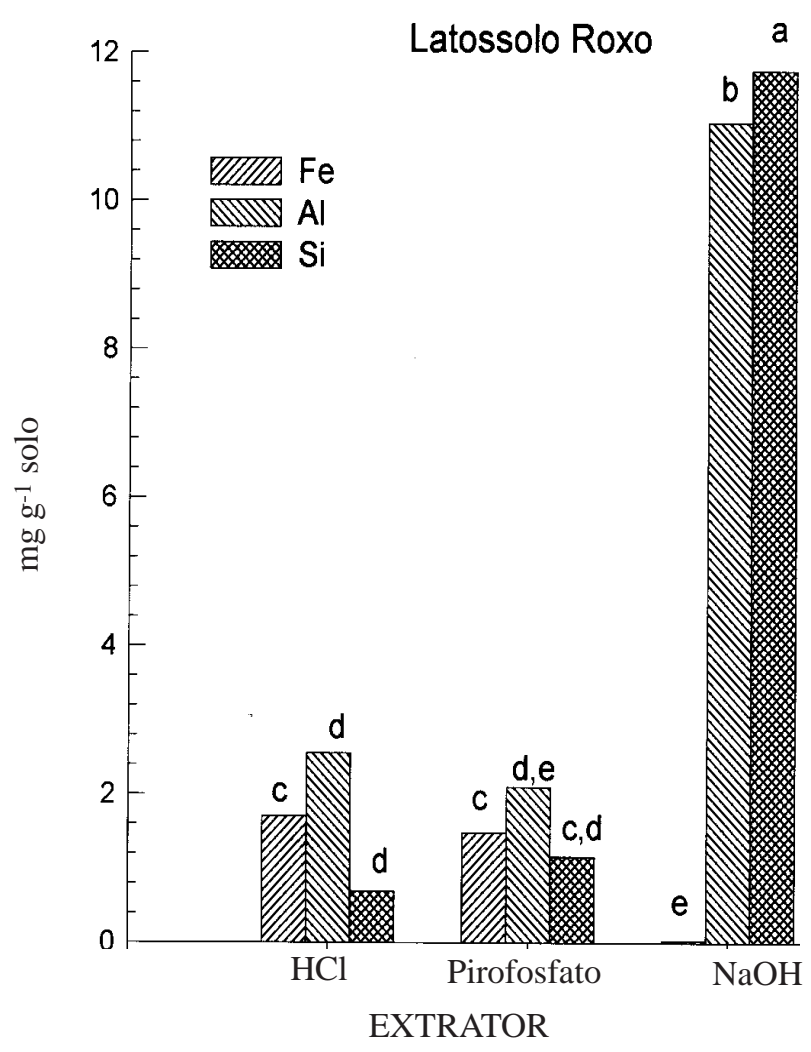

Figura 1. Teores de Fe, Al e Si, determinados nos extratos de $\mathrm{HCl}$ 0,5 mol L-1, pirofosfato 0,15 $\mathrm{mol} \mathrm{L}^{-1}$ (pH 7) e NaOH 0,5 mol L-1 do método 2, no LR. Letras minúsculas diferenciam médias, pelo teste de Duncan a 5\%, para cada elemento em todas as três amostras das figuras 1, 2 e 3.
Considerando que, no presente estudo, para os maiores valores de $\mathrm{Fe}$, Al e Si extraídos com pirofosfato em $\mathrm{pH}$ neutro no solo $\mathrm{B}$ foi observado maior teor de $\mathrm{C}_{\mathrm{SH}}$ (Quadro 2) e que, em pH 7, os elementos $\mathrm{Fe}$, Al e Si não são solúveis na forma catiônica hidratada, infere-se que as formas solubilizadas nesta etapa estavam realmente associadas à matéria orgânica. Essa associação pode ser tanto na forma coordenada (Fe e Al) como na forma de géis de oxidróxidos ( $\mathrm{Fe}, \mathrm{Al}$ e $\mathrm{Si}$ ) e, ou, caulinita ( $\mathrm{Al}$ e $\mathrm{Si}$ ) de baixa cristalinidade que estavam adsorvidos na molécula orgânica. A razão molar obtida para $A l_{p} / S_{p}$ no solo $B$ foi de 0,67 , ao passo que no sol o LR foi de 1,9. Esse comportamento diferenciado mostra que o alumínio e o silício coextraídos devem ser provenientes de outras fontes, além da caulinita adsorvida na matéria orgânica. Tais resultados indicam a importância do Fe, $\mathrm{Al}$ e Si na interação tipo ponte (seja na forma de complexos como de géis de hidróxidos), sendo esse mecanismo mais rel evante para o solo $B$.

$\mathrm{Na}$ etapa alcalina, o teor de Fe determinado foi irrelevante nos dois solos, o que mostra que a presença de oxidróxidos deFe dispersos neste extrato foi desprezível. No LR, o Al e o Si foram extraídos

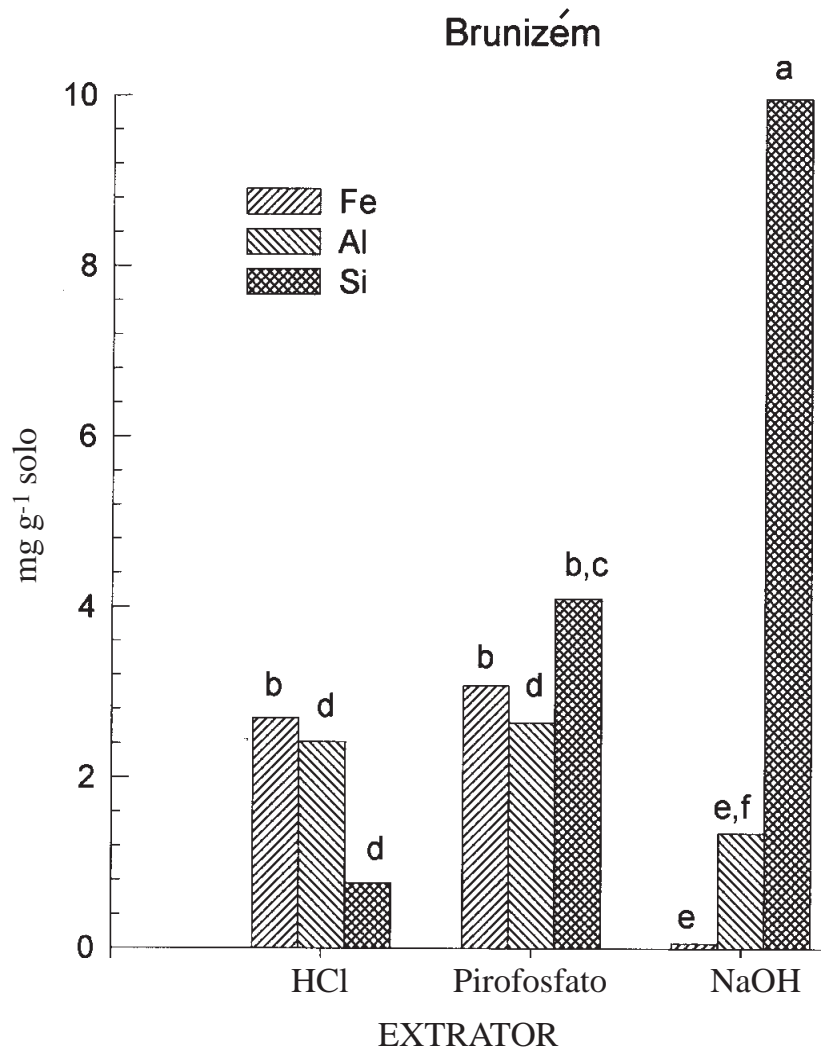

Figura 2. Teores de Fe, Al e Si, determinados nos extratos de $\mathrm{HCl} 0,5 \mathrm{~mol} \mathrm{~L}^{-1}$, pirofosfato $0,15 \mathrm{~mol} \mathrm{~L}^{-1}$ (pH 7) e NaOH 0,5 mol L-1 do método 2, no B. Letras minúsculas diferenciam médias, pelo teste de Duncan a 5\%, para cada elemento em todas as três amostras das figuras 1, 2 e 3. 
em quantidades próximas à razão molar 1:1 (Figura 1), indicando que caulinita adsorvida na SH foi solubilizada junto com a matéria orgânica. J á no B, a razão molar Si:Al foi de aproximadamente 7:1 e, neste caso, oSi solubilizado deve ter sido originário de outras fontes, além da eventual caulinita adsorvida. Provavelmente, a solução alcalina deve ter solubilizado Si de minerais no solo B.

$\mathrm{No} \mathrm{LO}$, o $\mathrm{HCl}$ extraiu quantidades elevadas de Fe e Al (Figura 3), que devem ser provenientes das impurezas inorgâni cas incorporadas ao lodo durante o processo de produção da cel ul ose. A solução neutra de pir ofosfato extraiu teores de $\mathrm{Fe}$, $\mathrm{Al}$ e Si inferiores aos obtidos nos dois solos. Na extração al cal ina, o Si solubilizado pode ter sido proveniente de constitutintes contidos na própria madeira.

\section{Análise elementar dos AH}

$\mathrm{Na}$ correção dos valores de $\mathrm{C}, \mathrm{H}$ e $\mathrm{N}$ para uma baselivre decinzas, a eventual presença de minerais que se decompõem em temperaturas inferiores a $750^{\circ} \mathrm{C}$ (ex.: goethita e caulinita) poderia levar à superestimação do teor de cinzas determinado nos $\mathrm{AH}$, especialmente nas amostras extraídas com $\mathrm{NaOH}$. Porém, no presente estudo, esta contribuição foi relevada, considerando a abundância do material orgânico na fração de ácidos húmicos purificados (>90\%).

$\mathrm{O} A \mathrm{H}$ extraído com $\mathrm{NaOH}$ no método 1 no LR apresentou composição el ementar, caráter aromático $(H / C=1,1)$ e grau de oxigenação $(O / C=0,58)$ semelhantes aos obtidos para o AH extraído pelo mesmo método no solo B (Quadro 4). Uma vez que não houve extração de $A H$ na etapa com pirofosfato no método 2 no LR, não foi realizada a análise do $\mathrm{AH}$ da etapa alcalina deste método.

No solo $B$, o extrator pirofosfato solubilizou $A H$ com menor teor de $\mathrm{C}, \mathrm{H}$ eN emaior teor de $\mathrm{O}$, quando comparado com oAH solubilizado pel a etapa al calina do método 2. Os dados das razões calculadas mostram que o AH solúvel em pirofosfato é mais oxigenado, mais pobre em $\mathrm{N}$ e apresenta maior

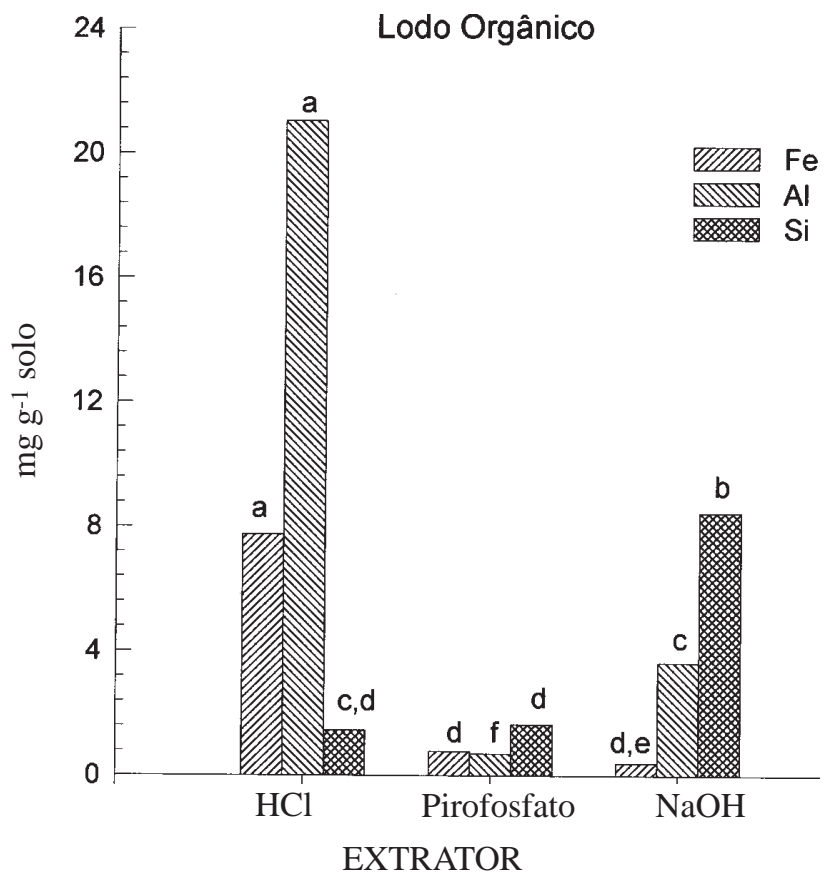

Figura 3. Teores de Fe, Al e $\mathrm{Si}$, determinados nos extratos de $\mathrm{HCl} 0,5 \mathrm{~mol} \mathrm{~L}^{-1}$, pirofosfato $0,15 \mathrm{~mol} \mathrm{~L}^{-1}\left(\mathrm{pH}\right.$ 7) e $\mathrm{NaOH} 0,5 \mathrm{~mol} \mathrm{~L}^{-1}$ do método 2 , no LO. Letras minúsculas diferenciam médias, pelo teste de Duncan a $5 \%$, para cada elemento em todas as três amostras das figuras 1,2 e 3.

caráter aromático do que o $\mathrm{AH}$ solubilizado por $\mathrm{NaOH}$. Resultados semelhantes foram obtidos por Schnitzer \& Schuppli (1989) e Hayes et al. (1996). No solo $B$, as amostras de AH solubilizadas com $\mathrm{NaOH}$ no método 2 e no método 1 apresentaram razão $\mathrm{C} / \mathrm{N}$ e caráter aromático similares. Esses resultados indicam que o extrator alcalino solubilizou SH quimicamente semel hantes.

As amostras deAH dolodo orgânicoapresentaram, em geral, valores mais el evados para os teores de H

Quadro 4. Teores de C, H, N e O (mg g $\left.{ }^{-1} \mathrm{AH}\right)$ nos ácidos húmicos, razão C/N e razões atômicas H/C e O/C

\begin{tabular}{|c|c|c|c|c|c|c|c|c|c|}
\hline Amostra & Método & Etapa & C & H & $\mathbf{N}$ & 0 & $\mathrm{C} / \mathrm{N}$ & $\mathrm{H} / \mathrm{C}$ & O/C \\
\hline LR & 1 & $\mathrm{NaOH}$ & 514 bc & $46 c$ & $39 \mathrm{~b}$ & $401 \mathrm{c}$ & $13 \mathrm{bcd}$ & $1,1 \mathrm{c}$ & $0,58 \mathrm{bc}$ \\
\hline B & $\begin{array}{l}1 \\
2\end{array}$ & $\begin{array}{l}\mathrm{NaOH} \\
\text { Pirofosfato } \\
\mathrm{NaOH}\end{array}$ & $\begin{array}{l}526 \mathrm{~b} \\
450 \mathrm{~d} \\
488 \mathrm{c}\end{array}$ & $\begin{array}{l}44 c \\
28 d \\
43 c\end{array}$ & $\begin{array}{l}36 \mathrm{~b} \\
22 \mathrm{c} \\
35 \mathrm{~b}\end{array}$ & $\begin{array}{l}395 \mathrm{c} \\
501 \mathrm{a} \\
434 \mathrm{~b}\end{array}$ & $\begin{array}{l}15 \mathrm{~b} \\
21 \mathrm{a} \\
14 \mathrm{bc}\end{array}$ & $\begin{array}{l}1,0 \mathrm{c} \\
0,75 \mathrm{~d} \\
1,0 \mathrm{c}\end{array}$ & $\begin{array}{l}0,56 \mathrm{c} \\
0,84 \mathrm{a} \\
0,68 \mathrm{~b}\end{array}$ \\
\hline LO & $\begin{array}{l}1 \\
2\end{array}$ & $\begin{array}{l}\mathrm{NaOH} \\
\text { Pirofosfato } \\
\mathrm{NaOH}\end{array}$ & $\begin{array}{l}562 a \\
504 b c \\
566 a\end{array}$ & $\begin{array}{l}67 \mathrm{a} \\
56 \mathrm{~b} \\
71 \mathrm{a}\end{array}$ & $\begin{array}{l}51 \mathrm{a} \\
32 \mathrm{~b} \\
58 \mathrm{a}\end{array}$ & $\begin{array}{l}321 d \\
409 \mathrm{bc} \\
304 \mathrm{~d}\end{array}$ & $\begin{array}{l}11 \mathrm{~cd} \\
16 \mathrm{~b} \\
9,9 \mathrm{~d}\end{array}$ & $\begin{array}{l}1,4 \mathrm{ab} \\
1,3 \mathrm{~b} \\
1,5 \mathrm{a}\end{array}$ & $\begin{array}{l}0,44 \mathrm{~d} \\
0,61 \mathrm{bc} \\
0,40 \mathrm{~d}\end{array}$ \\
\hline
\end{tabular}

Letras minúsculas, na coluna, diferenciam médias, pelo teste de Duncan a 5\%, para todas as três amostras. 
e $\mathrm{N}$, quando comparados com os respectivos $\mathrm{AH}$ de solos. Conseqüentemente, a razão $\mathrm{C} / \mathrm{N}$ foi menor nos $\mathrm{AH}$ da amostra LO, que apresentaram caráter mais alifático. Os valores mais baixos da razão O/C mostram que os $\mathrm{AH}$ do lodo orgânico apresentaram menor grau de oxigenação, quando comparados com os AH de solos. De maneira análoga ao observado no solo $B$, o pir ofosfato solubilizou $A H$ mais pobre em $\mathrm{N}$ e mais oxigenado do que o extrator al calino.

\section{CONCLUSÕES}

1. Os dois extratores empregados solubilizaram quantidades e proporções diferentes de substâncias húmicas. Tal comportamento diferenciado está relacionado com o mecanismo extrator de cada método e com a mineralogia do solo submetido à extração.

2. As interações de substâncias húmicas no solo de carga variável ocorreram principal menteatravés de reações de troca de ligantes e pontes de hidrogênio. J á no solo com predomínio de carga permanente, a interação via ponte de cátions coordenados ou de oxidróxidos também se mostrou importante.

3. No lodo orgânico, a introdução da etapa com pirofosfato (pH 7) não contribuiu para aumentar a quantidade solubilizada de substâncias húmicas. Nesta amostra, as impurezas inorgânicas contribuíram para as pontes catiônicas, e as pontes de hidrogêni o constituíram também mecanismos de interação importantes entre as moléculas de SH.

4. Os agentes extratores, empregados mostraramse seletivos para $\mathrm{SH}$ quimica e estruturalmente distintas. O extrator pirofosfato solubilizou no solo $B, A F$ com estrutura mais condensada/maior tamanho molecular do que os extraídos com $\mathrm{NaOH}$, conforme indicação da razão $\mathrm{E}_{350} / \mathrm{E}_{550}$. Os $\mathrm{AH}$ solubilizados pel o pirofosfato foram mais pobres em $\mathrm{N}$ e apresentaram grau de oxigenação e caráter aromático superiores aos verificados para $\mathrm{AH}$ extraídos com solução al cal ina.

\section{AGRADECIMENTOS}

Fapergs e CNPq.

\section{LITERATURA CITADA}

ALBUQUERQUE, J.A. Suscetibilidade de solos do Rio Grande do Sul a erosão entressulcos. Porto Alegre, Universidade Federal do Rio Grande do Sul, 1998. 156p. (Tese de Doutorado)
BAYER, C. Dinâmica da matéria orgânica no solos em diferentes sistemas de manejo. Porto Alegre, Universidade Federal do Rio Grande do Sul, 1996. 240p. (Tese de Doutorado)

BLOOM, P.R. \& LEENHEER, J.A. Vibrational, electronic and high-energy spectroscopic methods for characterizing humic substances. In: HAYES, M.H.B.; MACCARTHY, P.; MALCOLM, R.L. \& SWIFT, R.L., eds. Humic substances II: In search of structure. Chichester , J ohn Wiley, 1989. p.411-444.

BORGGAARD, O.K. Phase identification by selective dissolution techniques. In: STUCKI, J.W.; GOODMAN, B.A. \& SCHWERTMANN, U., eds. I ron in soils and clay minerals. Dordrecht, D. Reidel Publishing Company, 1988. p.83-98.

BURBA, P.; SHKINEV,V. \& SPIVAKOV, B.Y. On-linefractionation and characterization of aquatic humic substances by means of sequential-stage ultrafiltration. Fresenius J . Anal. Chem., 351:74-82, 1995

CALDERONI, G. \& SCHNITZER, M. Effects of age on the chemical structure of paleosol humic acids. Geochim. Chosmochim. Acta, 48:2045-2051, 1984

CHEN, Y; SENESI, N. \& SCHNITZER, M. I nformation provided on humic substances by $E_{4} / E_{6}$ ratio. Soil Sci. Soc. Am. J., 41:352-358, 1977.

CORNEJ O, J. \& HERMOSIN, M.C. Interaction of humic substances and soil clays. In: PICCOLO, A., ed. Humic substances in terrestrial ecosystems. Amsterdam, Elsevier, 1996. p.595-624.

DICK, D.P.; DICK,Y.P.; SILVA, L.F.; ZORTEA, R.B. \& ESSIG, C. Caracterização de produtos de degradação de lodos orgânicos em diferentes tempos e meios de digestão. R. Bras. Ci. Solo. 21:1-8, 1997.

GHOSH, K. \& SCHNITZER, M. Macromolecular structures of humic substances. Soil Sci., 129:266-276, 1980.

GU, B.; SCHMITT, J .; CHEN, Y.; LANG, L. \& MaCCARTHY , J . F. Adsorption and desorption of natural organic matter on iron oxides: mechanism and models. Environ. Sci. Technol., 28:38-46, 1994.

HAYES, T.M.; HAYES, M.H.B.; SKJ EMSTAD, J .O.; SWIFT, R.S. $\&$ MALCOLM, R.L. I solation of humic substances from soil using aqueous extractants of different $\mathrm{pH}$ andXAD resins, and their characterization by ${ }^{13}$ C-NMR.. In: CLAPP, C.E., ed. Humic substances and organic matter in soil and water environments: Characterization, transformations and interactions. Birmingham, IHSS, 1996. p.13-24.

KAISER, K. \& ZECH, W. Deffects in estimation of aluminum in humus complexes of podzolic soils by pyrophosphate extraction. Soil Sci., 161:452-458, 1996.

PARFITT, R.L.; FRASER, A.R. \& FARMER, V.C. Adsorption on hydrous oxides. III. Fulvic acid and humic acid on goethite, gibbsite and imogolite. J.Soil Sci., 28:289-296, 1977.

PARFITT, R.L. \& CHILDS, C.W. Estimation of forms of Fe and Al: a review, and analysis of contrasting soils by dissolution and moessbauer methods. Aust.J . Soil Res., 26:121-144, 1988.

RISTORI, G.G.; SPARVOLI, E.; NOBILI, M.\& D'ACQUI, L.P. Characterization of organic matter in particlesize fractions of Vertisolos. Geoderma, 54:295-305, 1992. 
SCHNITZER, M. \& SCHUPPLI, P. Extraction of organic matter from selected soils and particlesize fractionation with $0.5 \mathrm{M}$ and $0.01 \mathrm{M} \mathrm{Na}_{4} \mathrm{P}_{2} \mathrm{O}_{7}$ solutions. Can.J . Soil Sci., 69:253-262, 1989.

SCHUPPLI. P.; ROSS, G.J . \& MCKEAGUE, J .A. The effective removal of suspended materials from pyrophosphate extracts of soils from tropical and temperate regions. Soil Sci. Soc. Am. J ., 47:1026-1032, 1983.

SENESI, N.; MIANO,T.M. \& BRUNETTI, G. Methods and related problems for sampling soil and sediment organic matter. Extraction, fractionation and purification of humic substances. Quim. Anal., 13:26-33, 1994.

SINGER, A. \& HUANG, P.M. Humic acid effect on aluminium interlayering in montmorillonite. Soil Sci. Soc. Am. J., 57:271-279, 1993.
STEVENSON, F.J . Humus chemistry. Genesis, composition, reactions. 2.ed. New York, J ohn Wiley, 1994. 443p.

SWIFT, R.S. Organic matter characterization. In: SPARKS, D. L., ed. Methods of soil Analysis. Part 3. Chemical MethodsSSSA. Madison, SSSA, 1996. p.1001-1069. (Book series, 5)

TAN, K.H.; LOBARTINI, J.C.; HIMMELSBACH, D.S. \& ASMUSSEN, L.E. Composition of humic acids extracted under air and nitrogen atmosphere. Comm. Soil Sci. Plant Anal., 22:861-877, 1991.

TINSLEY, J. Determination of organic carbon in soils by dichromate mixtures. In: INTERNATIONAL CONGRESS OF SOIL SCIENCE, 4. Amsterdam, 1950. Transactions. Amsterdam, 1950. v.1. p.161-164.j

VARADACHARI, C.; MONDAL, A.H.\& GHOSH, K. Theinfluence of crystal edges on clay humus complexation. Soil Sci., 159:185-190, 1995. 